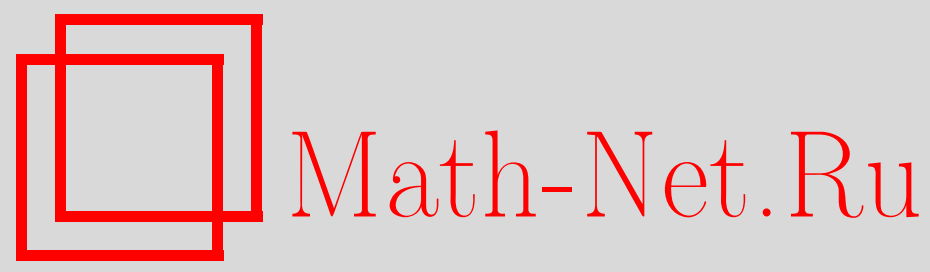

В. А. Исковских, Простое доказательство нерациональности трехмерной квартики, Матем. заметки, 1999, том 65, выпуск 5, 667-673

DOI: https://doi.org/10.4213/mzm1098

Использование Общероссийского математического портала Math-Net.Ru подразумевает, что вы прочитали и согласны с пользовательским соглашением http://www . mathnet.ru/rus/agreement

Параметры загрузки:

IP: 3.91 .87 .62

26 апреля 2023 г., 16:12:39

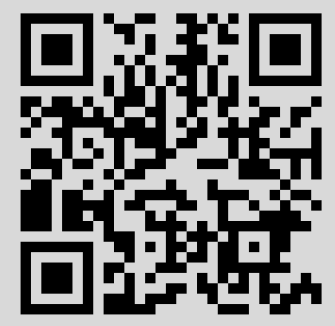




\section{ПРОСТОЕ ДОКАЗАТЕЛЬСТВО НЕРАЦИОНАЛЬНОСТИ ТРЕХМЕРНОЙ КВАРТИКИ}

\section{В. А. Исковских}

Передоказывается теорема о жесткости гладкой трехмерной квартики [1], следуя идее Корти [2], с использованием теоремы связности Шокурова.

Библиограффия: 8 названий.

1. Пусть $X=X_{4} \subset \mathbb{P}^{4}$ - гладкая гиперповерхность степени 4 (трехмерная квартика) над алгебраически замкнутьп полем характеристики 0. Довольно трудное доказательство нерациональности (точнее, бирациональной жесткости) $X$, данное в [1], до недавнего времени практически не претерпевало изменений. Некоторое упрощение в том же круге идей было предложено Пухликовым в [3] (и обобщено на высшие размерности в [4]). Недавно Корти [2] дал существенно более простое доказательство в рамках теории Мори. В этой заметке мы воспроизводим, по существу, доказательство Корти, используя только теорему обращения в нуль Каваматы-Фивега и некоторыепростые утверждения из [1] (включая, разумеется, неравенство Нетера-Фано).

Напомним утверждение о (сверх)жесткости $X$.

Теорема 1 [1], [2]. Не существует бирациональных отображсний, не являющихся проективными изоморфизмами, квартики ни на какое другое трехмерное многообразие Фано (и даже Мори расслоенное пространство). В частности, $X$ не рачионально.

2. Главная идея доказательства теоремы в [1], [2] и в этой заметке- существование неизоморфного бирационального отображения приводится к противоречию с неравенством Нетера-Фано. Сделаем это в несколько шагов.

ШАГ 1. (Неравенство Нетера-Фано). Предположим, что существует бирациональное отображение $\chi: X \rightarrow X^{\prime}$. Выберем очень обильную линейную систему $\mathcal{H}^{\prime}$ на $X^{\prime}$ и обозначим через $\mathcal{H}=\mathcal{H}_{X}=\chi_{\mathrm{pr}}^{-1}\left(\mathcal{H}^{\prime}\right)-$ собственный прообраз $\mathcal{H}^{\prime}$ на $X$. Тогда $\mathcal{H} \subset\left|-n K_{X}\right|, n \in \mathbb{Z}, n \geqslant 1$, не имеет неподвижных компонент, $\operatorname{dim} \mathcal{H}=\operatorname{dim} \mathcal{H}^{\prime}$. Пусть

$$
\begin{aligned}
\sigma: Y & =X_{N} \stackrel{\sigma_{N, N-1}}{\longrightarrow} X_{N-1} \longrightarrow \cdots \stackrel{\sigma_{i+1, i}}{\longrightarrow} X_{i} \stackrel{\sigma_{i, i-1}}{\longrightarrow} X_{i-1} \longrightarrow \cdots \stackrel{\sigma_{1,0}}{\longrightarrow} X_{0}=X \\
\sigma & =\sigma_{1,0} \cdots \sigma_{N, N-1}
\end{aligned}
$$

Работа выполнена при поддержке Российского фонда фундаментальных исследований, Фонда поддержки ведущих научных школ, проект № 96-15-96146 и INTAS-OPEN-97-2072. 
разрешение базисных множеств $\mathcal{H}$ по Хиронаке, т.е. с помошью последовательности раздутий $\sigma_{i, i-1}: X_{i} \rightarrow X_{i-1}$ с неособыми центрами $B_{i-1} \subset X_{i-1}, \operatorname{dim} B_{i-1}=0$ или 1. Тогда

$$
\mathcal{H}_{Y}:=\sigma_{\mathrm{pr}}^{-1}(\mathcal{H}) \sim \sigma^{*}(\mathcal{H})-\sum_{i=1}^{N} \nu_{i} \sigma_{N, i}^{*}\left(E_{i}\right),
$$

где $\mathcal{H}_{Y}-$ собственный прообраз $\mathcal{H}$ на $Y, \sigma_{N, i}=\sigma_{i+1, i} \cdots \sigma_{N, N-1}, E_{i}=\sigma_{i, i-1}^{-1}\left(B_{i-1}\right)$ - исключительные дивизоры, $\nu_{i} \geqslant 0$ - целые кратности базисных множеств $B_{i-1}, \nu_{1} \geqslant$ $\nu_{2} \geqslant \cdots \geqslant \nu_{N}$. Для канонических дивизоров имеем

$$
K_{Y}=\sigma^{*}\left(K_{X}\right)+\sum_{i=1}^{N} \delta_{i} \sigma_{N, i}^{*}\left(E_{i}\right), \quad \delta_{i}=2-\operatorname{dim} B_{i-1}
$$

Запишем

$$
n K_{Y}+\mathcal{H}_{Y}=\sigma^{*}\left(n K_{X}+\mathcal{H}_{X}\right)+\sum_{i=1}^{N}\left(n \delta_{i}-\nu_{i}\right) \sigma_{N, i}^{*}\left(E_{i}\right) .
$$

Перепишем (3) в виде

$$
n K_{Y}+\mathcal{H}_{Y}=\sigma^{*}\left(n K_{X}+\mathcal{H}_{X}\right)+\sum_{j=1}^{N}\left(\sum_{i \leqslant j} r_{j i}\left(\delta_{i} n-\nu_{i}\right)\right) F_{j}
$$

где $F_{j}=\sigma_{\mathrm{pr}}^{-1}\left(E_{j}\right)$ - собственные прообразы дивизоров $E_{j}, r_{j i}$ - целые неотрицательные числа, определяемые из однозначных разложений

$$
\sigma^{*}\left(E_{i}\right)=F_{i}+\sum_{j>i} r_{j i} F_{j}, \quad i, j=1, \ldots, N
$$

Числа $r_{i j}$ легко вычисляются индуктивно по разрешению

$$
r_{i i}=1, \quad r_{j i}=\sum_{k: B_{j-1} \subset F_{k}} r_{k i}, \quad j>i, \quad k \geqslant i, \quad k \leqslant j-1
$$

(для простоты через $F_{k}$ мы будем обозначать также любой его образ $\sigma_{N, l}\left(F_{k}\right)=$ $\left(\sigma_{l k}^{-1}\right)_{\mathrm{pr}}\left(E_{k}\right) \subset X_{l}, l \geqslant k$, в частности, $F_{k}=E_{k}$ при этом соглашении). Так как $\sum F_{i}$ - дивизор с нормальньми пересечениями, число слагаемых в (6) не превосходит $\delta_{j}+1$, т.е. $\leqslant 2$, если $B_{j-1}-$ кривая, и $\leqslant 3$, если $B_{j-1}-$ точка.

ЛЕмма 1 (Неравенства Нетера-Фано, см. например, [1], [5], [6]). Если ұ не изоморфизм, то $n \geqslant 2$ и линейная система $\mathcal{H}$ обладает максимальной особенностью, т.е. в каждом из выражений (3) и (4) найдется $E_{i}$, соответственно $F_{j}$, такие, что

$$
\begin{aligned}
n \delta_{i}-\nu_{i} & <0 \\
\sum_{i \leqslant j} r_{i j}\left(n \delta_{i}-\nu_{i}\right) & <0 .
\end{aligned}
$$


Отметим, что неравенства Нетера-Фано имеют место в подходящей формулировке для произвольных Мори расслоенных пространств (см. [6]).

ШАГ 2. (Исключение максимальных особенностей: простые случаи). Предположим, что существует максимальная особенность, скажем $F_{j}$ с неравенством $(8)$. Тогда существует индекс $i \leqslant j$, для которого выполняется неравенство $(7)$ и $r_{j i} \neq 0$ и, следовательно, $\sigma_{j, i}\left(F_{i}\right) \subset E_{i}$. Пусть $C=\sigma_{j, 0}\left(F_{i}\right) \subset X$ - центр максимальной особенности и $\nu(C)-$ кратность $C$ в базисном множестве Bs $\mathcal{H}$. Тогда $\nu(C) \geqslant \nu_{i}>\delta_{i} n_{i}$, так как $C \subset \sigma_{i, 0}\left(E_{i}\right)$.

Возможны три случая:

(i) $C=P$ - точка кратности $\nu(P)>2 n$;

(ii) $C$ - кривая кратности $\nu(C)>n$;

(iii) (бесконечно близкий случай) $C=P$ - точка кратности $\nu(P)>n, B_{i-1}-$ кривая c $\nu_{i}>n$.

В нашем случае гладкой трехмерной квартики $X$ простые рассуждения, восходящие к Фано, исключают первые два случая.

Лемма $2([1,5.2]) . \nu(P)<2 n$ для любой точки $P \in \operatorname{Bs} \mathcal{H} u \nu(C) \leqslant n$ для любой кривой $C \subset \mathrm{Bs} \mathcal{H}$.

Таким образом, задача сводится к исключению трудного случая (iii). Для этого мы привлечем идею Корти: ограничим (4) на общее гиперплоское сечение $S \ni P$ и воспользуемся теоремой связности Шокурова. Это локальный вопрос в окрестности точки $P=\sigma_{j, 0}\left(F_{i}\right)$ - центра максимальной особенности, и то, что $X-$ квартика, не будет использоваться до заключительного шага 3.

Итак, мы будем предполагать, что в окрестности точки $P \in X$ мы имеем разрешение (1) с $B_{0}=P, \nu_{1}=\nu(P), E_{1}=\sigma_{1,0}^{-1}(P)$ и т.д. и что существует бесконечно близкая максимальная особенность $F_{j}$ с $\sigma_{j, 0}\left(F_{j}\right)=P$. Пусть $S$ - общее гиперплоское сечение (росток гладкой поверхности), проходящее через $P$. Тогда

$$
S_{Y}:=\sigma_{\mathrm{pr}}^{-1}(S) \sim \sigma^{*} S-\sigma_{N, 1}^{*}\left(E_{1}\right)=\sigma^{*} S-\sum_{j=1}^{N} r_{j 1} F_{j} .
$$

Для того чтобы при ограничении $(4)$ на $S_{Y}$ получить формулу такого же типа, ограничим вместо (4) следующее выражение

$$
\begin{aligned}
n\left(K_{Y}+S_{Y}\right)+\mathcal{H}_{Y}= & \sigma^{*}\left(n K_{X}+n S+\mathcal{H}_{X}\right) \\
& +\sum_{j=1}^{N}\left(\sum_{i \leqslant j} r_{i j}\left(\delta_{i} n-\nu_{i}\right)-r_{j 1} n\right) F_{j} .
\end{aligned}
$$

Неравенство для максимальной особенности в (8) запишется теперь в виде

$$
c_{j}:=\sum_{i \leqslant j} r_{i j}\left(\delta_{i} n-\nu_{i}\right)-r_{j 1} n<-n,
$$

так как $r_{j 1} \geqslant 1$, поскольку $\sigma_{i, 0}\left(F_{j}\right)=P=B_{0}$. Ограничивая $(9)$ на общую поверхность $S \ni P$, получаем

$$
n K_{S_{Y}}+\left.\mathcal{H}_{Y}\right|_{S_{Y}}=\sigma^{*}\left(n K_{S}+\mathcal{H}_{S}\right)+\sum_{j=1}^{N} c_{j} F_{j} \mid S_{Y} .
$$

Следующая лемма - самый существенный момент доказательства - является следствием теоремы связности Шокурова, которая выводится из теоремы обращения в нуль Каваматы-Фивега для бирационального морфизма $\sigma: Y \rightarrow X$. 
ЛЕмма 3. Предположим, что существует $F_{j} c c_{j} \leqslant-n$. Тогда найдется $F_{a} c$ $c_{a} \leqslant-n u F_{a} \cap S_{Y} \neq \varnothing$.

ДокАЗАТЕЛЬСтво. Разделим (10) на $n$ и запишем в виде

$$
\begin{aligned}
K_{Y} & \equiv \sigma^{*}\left(K_{X}+S+\frac{1}{n} \mathcal{H}_{X}\right)-\frac{1}{n} \mathcal{H}_{Y}-S_{Y}+\sum_{j=1}^{N} \frac{1}{n} c_{j} F_{j} \\
& =\sigma^{*}\left(K_{X}+S+\frac{1}{n} \mathcal{H}_{X}\right)+A-B,
\end{aligned}
$$

где $A, B-\mathbb{Q}$ - дивизоры и

$$
B=S_{Y}-\sum_{c_{j} \leqslant-n} \frac{c_{j}}{n} F_{j} .
$$

Покажем, следуя Шокурову (см. например, $[7,7.4],[8,17.4])$, что $\operatorname{Supp} B=S_{Y}+$ $\sum_{c_{j} \leqslant-n} F_{j}$ связен в окрестности слоя $\sigma^{-1}(P)$. Для этого перепишем $(12)$ в виде, удобном для применения теоремы обращения в нуль

$$
\ulcorner A\urcorner-\llcorner B\lrcorner \equiv K_{Y}+\left(-\sigma^{*}\left(K_{X}+S+\frac{1}{n} \mathcal{H}_{X}\right)\right)+\{-A\}+\{B\},
$$

где, как обычно, для $\mathbb{Q}$ дивизора $D=\sum d_{i} D_{i}, d_{i} \in \mathbb{Q},\llcorner D\lrcorner=\sum\left\llcorner d_{i}\right\lrcorner D_{i},\ulcorner D\urcorner=$ $\sum\left\ulcorner d_{i}\right\urcorner D_{i}, \quad\{D\}=\sum\left\{d_{i}\right\} D_{i}$ обозначает нижнюю, верхнюю целую часть и дробную часть соответственно. Отметим, что $\operatorname{Supp} B=\operatorname{Supp}\llcorner B\lrcorner$, дивизор $\ulcorner A\urcorner$ эффективен и составлен из исключительных дивизоров, поскольку $\left\ulcorner-\frac{1}{n} \mathcal{H}_{Y}\right\urcorner=0$, так как $n \geqslant 2$; $\{-A\},\{B\}$ - эффективные дробные дивизоры с нормальными пересечениями. Нормальность пересечений с $S_{Y}$ и $\mathcal{H}_{Y}$ следует из общности выбора $S \ni P$ и общего дивизора $H_{Y}$ свободной линейной системы $\mathcal{H}_{Y}$, нормальность пересечений исключительных дивизоров обеспечена выбором разрешения (1).

Применим $\sigma_{*}$ к точной последовательности

$$
0 \rightarrow \mathcal{O}_{Y}(\ulcorner A\urcorner-\llcorner B\lrcorner) \rightarrow \mathcal{O}_{Y}(\ulcorner A\urcorner) \rightarrow \mathcal{O}_{\llcorner B\lrcorner}(\ulcorner A\urcorner) \rightarrow 0
$$

и заметим, что $\sigma_{*} \mathcal{O}_{Y}(\ulcorner A\urcorner)=\mathcal{O}_{X}$, так как $\ulcorner A\urcorner-$ эффективньй дивизор, составленный из исключительных дивизоров, и $R^{i} \sigma_{*} \mathcal{O}_{Y}(\ulcorner A\urcorner-\llcorner B\lrcorner)=0, i \geqslant 1$, по относительной теореме обращения в нуль Каваматы-Фивега для бирациональногоморфизма $\sigma: Y \rightarrow X$ (см. например $[7,2.17])$, надо заметить только, что $\left(-\sigma^{*}\left(K_{X}+S+\frac{1}{n} \mathcal{H}_{X}\right)\right)$ тривиален на слоях и поэтому численно эффективен и объемен на общем слое.

В результате получаем точную последовательность

$$
\sigma_{*} \mathcal{O}_{Y}(\ulcorner A\urcorner)=\mathcal{O}_{X} \rightarrow \sigma_{*} \mathcal{O}_{\llcorner B\lrcorner}(\ulcorner A\urcorner) \rightarrow 0 .
$$

Если $\llcorner B\lrcorner=B_{1}+B_{2}-$ несвязное объединение двух дивизоров в окрестности слоя $\sigma^{-1}(P)$, то мы имеем $\sigma_{*} O_{B}(\ulcorner A\urcorner)_{P}=\sigma_{*} O_{B_{1}}(\ulcorner A\urcorner)_{P}+\sigma_{*} O_{B_{2}}(\ulcorner A\urcorner)_{P}$, что невозможно, так как сумма двух ненулевых модулей не может быть фактором циклического модуля $\mathcal{O}_{X, P}$. Это доказьвает связность $\operatorname{Supp} B=S_{Y}+\sum_{c_{j} \leqslant-n} F_{j}$ и тем самым лемму, поскольку по условию существует $F_{j}$ с $c_{j} \leqslant-n$.

3. Рассмотрим теперь равенство (12) с учетом неравенства (10) и предыдущей леммы. Пусть $a$ - наименьший индекс такой, что $F_{a} \cap S_{Y} \neq 0$ и $c_{a} \leqslant n$. Введем следующие дополнительные обозначения:

$S_{i}=\left(\sigma_{i, 0}^{-1}\right)_{\mathrm{pr}}(S)$ - собственный прообраз на $X_{i}$ общего гиперплоского сечения $S \ni P$;

$\mathcal{L}_{i}=\mathcal{H}_{X_{i}} \mid S_{i}-$ собственный прообраз на $S_{i}$ линейной системы $\mathcal{L}_{0}=\mathcal{H}_{X} \mid S$. 
Лемма 4. i) $B$ предыдущих обозначениях $a=1 \Leftrightarrow \nu_{1} \geqslant 2 n$.

ii) $\bar{E}_{1}=E_{1} \cap S_{1}-$ прямая на $E_{1} \cong \mathbb{P}^{2}$; если $F_{i} \cap S_{Y} \neq 0, i>1$, mо $\sigma_{i, 1}\left(F_{i}\right)=$ $\sigma_{i, 1}\left(E_{i}\right) \subset E_{1} \cong \mathbb{P}^{2}-$ кривая и $E_{i}$ - линейчатая поверхность.

iii) $E c \wedge u r_{a i} \neq 0$, mo $E_{i} \cap S_{i} \neq 0$ и $\sigma_{a, 1}\left(F_{a}\right)=\sigma_{i, 1}\left(E_{i}\right)$ на $E_{1}$.

iv) $\mathcal{L}_{a}^{2} \leqslant \mathcal{L}_{0}^{2}-\sum_{i=1, r_{a i} \neq 0}^{a} \nu_{i}^{2}$.

ДокАЗАТЕЛЬСтво. і) Имеем $c_{1}=r_{11} n \delta_{1}-\nu_{1}-r_{11} n=n-\nu_{1}$ и, поскольку $E_{1} \cap$ $S_{1} \neq 0$, то $a=1$ равносильно $n-\nu_{1} \leqslant-n$.

ii) Если $i>1$ и $F_{i} \cap S_{Y} \neq 0$, то $\sigma_{N, 1}\left(F_{i} \cap S_{Y}\right)=\sigma_{N, 1}\left(F_{i}\right) \cap S_{1} \subset E_{1}$ не пусто; значит $\sigma_{N, 1}\left(F_{i}\right)$ - кривая, так как $S_{1} \cap E_{1}$ - общая прямая.

iii) Если $r_{a i} \neq 0$, то $\sigma_{a, i}\left(E_{a}\right) \subset E_{i}$ и $0 \neq \sigma_{a, i}\left(S_{a} \cap F_{a}\right) \subset S_{i} \cap \sigma_{a, i}\left(E_{a}\right) \subset S_{i} \cap E_{i}$. По ii) образы $F_{a}$ и $E_{i}$ на $E_{1}$ являются кривыми, содержашимися одна в другой; ввиду неприводимости они совпадают.

iv) Имеем

$$
\mathcal{L}_{a}^{2}=\sigma_{a, 0}^{*}\left(\mathcal{L}_{0}\right)^{2}-\sum_{i=1}^{a} d_{i} \nu_{i}^{2} \leqslant \mathcal{L}_{0}^{2}-\sum_{i=1, r_{a} \neq \neq 0}^{a} d_{i} \nu_{i}^{2} \leqslant \mathcal{L}_{0}^{2}-\sum_{i=1, r_{a i} \neq 0}^{a} \nu_{i}^{2},
$$

где $d_{i}=B_{i-1} \cdot S_{i-1}$ на $X_{i-1}$ для $i>1, d_{1}=1$. Отметим, что из-за обшности $S$ пересечения $B_{i-1} \cdot S_{i-1}$ можно считать трансверсальными.

ЗАМЕЧАнИЕ. В действительности, если $a>1$ и $r_{a i} \neq 0$, то $d_{i}=1$. Это следует из теоремы связности Шокурова для $\left(S, \mathcal{L}_{0}\right)$ : пересечение $F_{a} \cap S_{a}$ должно быть связным, и так как $a>1$, то $E_{a}$ - линейчатая поверхность (по лемме 3 ) и $F_{a} \cap S_{a}=E_{a} \cap S_{a}$ состоит из одного слоя. Значит, образ $F_{a}$ на $E_{1}$ и образы всех $F_{i}$ с $r_{a i} \neq 0$ являются одной и той же прямой; отсюда $d_{i}=B_{i-1} \cdot S_{i-1}=1$.

4. Дальнейшая цель - оценить снизу последнюю сумму в (14) при условии $c_{a} \leqslant-n$ и показать, что она достаточно большая (больше, чем $\mathcal{L}_{0}^{2}$ на квартике X).

Лемма 5. Если $а>>1$, mo $\sum_{i=1}^{a} \nu_{i}^{2}>4 n^{2}$.

ДокАЗАТЕльство. Для удобства перенумеруем последовательно $\left\{E_{i}, 1 \leqslant a \leqslant a \mid\right.$ $\left.r_{a i} \neq 0\right\}: E_{1}, E_{2}, \ldots, E_{b}, b=\#\left\{i \mid r_{a i} \neq 0\right\}$ и положим (в новых обозначениях) $r_{s i}=$ $r_{s+1-i}$. В этих обозначениях условие $c_{a} \leqslant-n$ запишется в виде

$$
r_{b} \nu_{1}+r_{b-1} \nu_{2}+\cdots+r_{1} \nu_{b} \geqslant\left(\sum_{i=1}^{b} r_{i}+1\right) n
$$

так как в старых обозначениях

$$
c_{a}=\sum_{i=1}^{a} r_{a i}\left(\delta_{i} n-\nu_{i}\right)-r_{a 1} n \leqslant-n
$$

и все слагаемые с $\delta_{i}=2$ равны нулю, кроме первого при $i=1$.

Имеем по определению $r_{j i}$ в (5)

$$
r_{1}=r_{2}=1, \quad r_{b} \geqslant r_{b-1} \geqslant \cdots \geqslant r_{1}=1, \quad r_{i+1} \leqslant r_{i}+r_{i-1} .
$$


Минимум квадратичной формы $\sum_{i=1, r_{a} \neq 0}^{a} \nu_{i}^{2}=\sum_{j=1}^{b} \nu_{j}^{2}$ (в новых обозначениях) при условии (15) находится стандартным способом:

$$
\min \left\{\sum_{j=1}^{b} \nu_{j}^{2} \mid \sum_{j=1}^{b} r_{j} \nu_{b+1-j} \geqslant \sum_{i=1}^{b}\left(r_{i}+1\right) n\right\}=\frac{\left(\sum_{j=1}^{b} r_{j}+1\right)^{2}}{\sum_{j=1}^{b} r_{j}^{2}} n^{2}
$$

Осталось показать, что $\left(\sum_{j=1}^{b} r_{j}+1\right)^{2}>4 \sum_{j=1}^{b} r_{j}^{2}$ при $b>1$. По индукции предположим, что это неравенство справедливо для $s<s+1 \leqslant b$. Запишем

$$
\begin{aligned}
\left(\sum_{j=1}^{s+1} r_{j}+1\right)^{2} & =r_{s+1}^{2}+2 r_{s+1}\left(\sum_{j=1}^{s} r_{j}+1\right)+\left(\sum_{j=1}^{s} r_{j}+1\right)^{2} \\
4 \sum_{j=1}^{s+1} r_{j}^{2} & =4 r_{s+1}^{2}+4 \sum_{j=1}^{s} r_{j}^{2}
\end{aligned}
$$

Отсюда достаточно показать, что $r_{s+1} \leqslant \frac{2}{3}\left(\sum_{j=1}^{s} r_{j}+1\right)$.

Опять по индукции

$$
\begin{aligned}
r_{s+1} & \leqslant r_{s}+r_{s-1} \leqslant r_{s}+\frac{2}{3}\left(\sum_{j=1}^{s-2} r_{j}+1\right) \\
& \leqslant \frac{2}{3} r_{s}+\frac{2}{3} r_{s-1}+\frac{2}{3}\left(\sum_{j=1}^{s-2} r_{j}+1\right)=\frac{2}{3}\left(\sum_{j=1}^{s} r_{j}+1\right)
\end{aligned}
$$

так как $r_{s} \leqslant 2 r_{s-1}$. Лемма доказана.

Теперь возвратимся к квартике Х и сделаем последний шаг.

ШАГ 3 (исключение бесконечно близкого случая). Покажем, что случай іiі) в шаге 2 не реализуется. Предположим, что максимальная особенность типа іiі) существует; тогда по (10) и лемме 2 сушествует $F_{a}$ с $c_{a} \leqslant-n$. Пусть $a-$ наименьший индекс с этим свойством; тогда $a>1$ по лемме $4 \mathrm{i}$ ), так как $\nu_{1}<2 n$ по лемме 1 . Осталось воспользоваться леммой 3 и леммой 4 , принимая во внимание, что $\mathcal{L}_{i}^{2}>0$, так как линейная система $\mathcal{L}_{i}$ не имеет неподвижных компонент из-за общности $S \ni P$ и не составлена из пучка,

$$
0<\mathcal{L}_{a}^{2} \leqslant 4 n^{2}-\sum_{i=1, r_{a i} \neq 0}^{a} \nu_{i}^{2} \leqslant 4 n^{2}-\frac{\left(\sum_{j=1}^{b} r_{j}+1\right)^{2}}{\sum_{j=1}^{b} r_{j}^{2}} n^{2}<0
$$

Это противоречие завершает доказательство теоремы. 


\section{СПИСОК ЦИТИРОВАННОЙ ЛИТЕРАТУРЫ}

[1] Исковских В. А., Манин Ю. И. Трехмерные квартики и контрпримеры к проблеме Люрота // Матем. сб. 1971. Т. 86(128). №1. С. 140-166.

[2] Corti A. Singularities of linear systems and 3-fold birational geometry. Preprint Univ. of Cambridge, 1998.

[3] Пухликов А. В. Замечание к теореме В.А. Исковских и Ю.И. Манина о трехмерной квартике // Тр. МИРАН. 1995. Т. 208. С. 278-289.

[4] Pukhlikov A. V. Birational automorphisms of Fano hypersurfaces // Invent. Math. 1998. V. 134. № 2. P. 401-426.

[5] Исковских В.А. Бирациональные автоморфизмы трехмерных алгебраических многообразий // Итоги науки и техники. Современные проблемы математики. Т. 12. М.: ВИНИТИ, 1979. C. $159-236$.

[6] Corti A. Factoring birational maps of 3-folds after Sarkisov // Journ. Alg. Geom. 1995. V. 4. P. 223-254.

[7] Kollar J. Singularities of pairs // Proc. Sympos. Pure Math. 1998. V. 62. P. 224-287.

[8] Kollar J. et al. Flips and abundance for algebraic threefolds // Asterisque. 1993. V. 211.

Математический институт им. В. А. Стеклова

Поступило

01.12 .98 